\title{
Identification of young people at risk of psychosis
}

\author{
Sophie Parker \& Shôn Lewis
}

\begin{abstract}
Operational criteria for detecting prodromal, or at-risk, mental states have been developed largely on the basis of individuals seeking help for attenuated or brief, self-limiting symptoms that do not meet threshold criteria for psychotic disorder. These individuals present largely to primary care and other non-specialist mental health settings. Follow-up studies have confirmed that $15-40 \%$ will make the transition to full psychosis within 12 months. Cognitive therapy alone or in combination with lowdose atypical antipsychotics has been shown to be efficacious in reducing or delaying the transition to psychosis, as well as in ameliorating the severity of non-psychotic symptoms and distress. Antipsychotic medication alone has not shown significant efficacy, but results are suggestive of some advantage from drug treatment. Further work is needed to clarify the relative merits of these interventions.
\end{abstract}

The notion of being able to prevent the onset of schizophrenia and other psychotic disorders by detecting and intervening in the prodromal phase has been a goal discussed for many years, by Harry Stack Sullivan in 1927, Ainslie Meares in 1959 and others. The potential benefits have been seen to include the prevention or minimisation of psychosocial (Johnstone et al, 1986; Loebel et al, 1992) and possibly biological disruption (Wyatt, 1991). In addition, there is evidence to suggest that the length of time a psychotic episode is left untreated is negatively correlated with an individual's prognosis (Crow et al, 1986; Loebel et al, 1992). This concept of a 'critical period' has had a significant impact on UK service provision for people diagnosed with a psychotic illness, in the provision of early intervention (Department of Health, 2001, 2003). If we can identify people who may be at risk of psychosis, we may also have the opportunity to reduce the duration of untreated psychosis.

\section{Identifying risk of psychosis: the Melbourne criteria}

Current research in this area began in Melbourne, Australia, with the work of Yung, McGorry and colleagues. In the 1990s two articles appeared in which they explained the rationale for the establishment of the Personal Assessment and Crisis Evaluation (PACE) clinic, aimed at monitoring and providing care for young, help-seeking people described as being at high risk for impending psychosis (Yung et al, 1996, 1998).

Yung and colleagues set about defining operationally a set of clinical features that precede a first psychotic episode (Phillips et al, 2000). The features identified comprise a combination of state and trait factors.

The state factors fall into two groups: attenuated symptoms and brief limited intermittent psychotic symptoms (BLIPS). Attentuated symptoms are lowgrade positive psychotic symptoms, for example hallucinatory experiences assessed at either the upper extremes of normal limits or unusual thoughts, such as ideas of reference or persecutory ideas that are easily dismissed and do not affect behaviour to a significant extent. Brief limited intermittent psychotic symptoms involve brief bursts of frank psychotic symptoms lasting less than a week and spontaneously resolving.

The trait-based criteria include a combination of trait factors of psychosis (a family history of psychotic disorder or the individual meeting diagnostic criteria for schizotypal personality disorder) and a change in mental state lasting at least 1 month and having a clear association with recent functional deterioration.

Sophie Parker is a trainee clinical psychologist at the University of Manchester (Division of Clinical Psychology, University of Manchester, 2nd Floor Zochonis Building, Brunswick Street, Manchester M13 9LP, UK. Email: spophs@ntlworld.com). Her particular interests lie in detection and intervention for young people at risk of developing psychosis and links between trauma and psychosis. Shôn Lewis is Professor of Adult Psychiatry in the University of Manchester's Education and Research Centre at the Wythenshawe Hospital Manchester. He has research interests in the causes and treatments of psychosis, especially early psychosis. 
Box 1 The Melbourne criteria: clinical features of the at-risk mental state

State-based criteria

- Attenuated symptoms: low-grade positive psychotic symptoms

- Brief limited intermittent psychotic symptoms (BLIPS): brief bursts of frank psychotic symptoms

Trait-based criteria

A combination of:

- trait factors of psychosis (family history of psychotic disorder or the individual meeting criteria for schizotypal personality disorder)

plus

- a change in mental state lasting at least 1 month and clearly associated with recent functional deterioration

For all these conditions (Box 1), the Melbourne team preferred the term 'at-risk mental state' to the more usual term 'prodromal', on the basis that only a proportion would go on to develop psychosis.

\section{Validation studies}

Using these state and trait factors, Yung's team recruited a group of help-seeking people whom they judged to be at high risk of transition to psychosis (Phillips et al, 2000). During the 24-month follow-up, $33 \%$ of the group developed psychosis. Following this pilot study, the PACE intake criteria were modified in an effort to increase the specificity of detection. In a subsequent study using the revised criteria, $41 \%$ (20/49) of participants became psychotic within 12 months, with a small number of additional transitions over an 'extended period' (Yung et al, 2003). The peak time of risk for developing psychosis was within 4.5 months of entry into the study. Thus, it appeared possible to identify individuals who are at risk of developing psychosis at rates onethousand-fold higher than in the general population. More recent studies have confirmed this, although transition rates are not quite so high: a later study completed at the PACE clinic reported that $28 \%$ $(29 / 104)$ of their sample had made transition by 6-month follow-up, rising to $35 \%(36 / 104)$ by $12-$ month follow-up (Yung et al, 2004).

Since these initial studies by the PACE team, a number of groups around the world have used the criteria established by Yung et al (the Melbourne criteria) to identify and provide varying interventions for this population. Clearly, the detection of false positives is a serious problem, and studies attempting therapeutic interventions run the risk of including a majority of people who will never receive a diagnosis of psychosis. The original Melbourne criteria have been operationalised in trials using a number of existing instruments, including the Positive and Negative Syndrome Scale (PANSS) and the Brief Psychiatric Rating Scale (BPRS). However, there are limitations to using tools that have been developed for a population who have existing psychosis, in terms of sensitivity for measuring developing symptoms. In addition, there are items relating to insight in both the PANSS and the BPRS that can be problematic and inappropriate for this client group. More recently, other tools specifically designed to assess this group of people have been developed. These include the Comprehensive Assessment of At-Risk Mental States (CAARMS; Yung et al, 2001) and the Structured Interview for Prodromal Symptoms and the Scale of Prodromal Symptoms (SIPS/SOPS; Miller et al, 1999; McGlashan et al, 2001).

The purpose of the Bonn Scale for the Assessment of Basic Symptoms (BSABS) is to identify individuals at an earlier stage of the prodrome on the basis of symptoms such as particular perceptual aberrations. Klosterkotter et al (2001) used the BSABS as a predictive instrument and reported that $70 \%$ of 110 participants who had endorsed one or more items on the BSABS had developed DSM-IV schizophrenia at 9.6-year follow-up. However, the participants in this study comprised people initially referred to a tertiary clinic on the grounds of possible incipient psychosis. Used in the general population its predictive power would be low: Warner (2002) has suggested that only about $2 \%$ of people identified as cases would go on to develop a psychotic disorder.

\section{The role of substance use in the at-risk population}

A number of studies have examined the overlap and relationship between substance use and psychosis, given that many of the symptoms experienced by people in the at-risk group parallel the effects of the use of a variety of substances. A review of substance use in 100 at-risk clients revealed that $18 \%$ met criteria for cannabis dependence in the previous 12 months (Phillips et al, 2002). The possible role that substances play in those who may be at increased risk of developing psychosis has been explored in a number of studies, primarily focused on cannabis use.

Miller et al (2001) found that cannabis and other illicit drug use predicted occurrence of psychotic 
symptoms in both at-risk clients (defined by genetic loading) and those in a control group. They observed a dose-response effect of drug use on subsequent likelihood of experiencing positive psychotic symptoms. Colleagues at the PACE clinic found that cannabis use did not significantly contribute to the risk of transition to psychosis within a 12month period (Phillips et al, 2002). Henquet et al (2005) conducted a prospective cohort study of 2437 randomly selected young people, identified using the 'paranoid ideation' and 'psychoticism' scales of the 90-item revised Symptom Checklist (SCL-90-R). They found a positive dose-response association between cannabis use around baseline and risk of psychosis at follow-up, and noted that this was significantly stronger in those identified as having a higher predisposition to psychosis at baseline. Verdoux et al (2003) assessed vulnerability to psychosis using the Community Assessment of Psychotic Experiences (CAPE), comparing high-risk individuals with people without a high predisposition for psychosis. They concluded that cannabis use may promote psychotic experiences in those with an existing vulnerability.

Although most of the above research is suggestive of the detrimental effects of substance misuse for both the at-risk and general populations, caution should be taken in light of the varying study methodologies. For a more comprehensive review of substance misuse in the at-risk group see Leicester (2006).

\section{Options for treatment: intervention studies}

A variety of interventions have been offered to those meeting criteria for being in an at-risk mental state. In their pilot study, Yung et al (1996) adopted a 'wait and see' approach. In a subsequent study conducted at the PACE clinic, Yung and colleagues offered a combination of supportive counselling, case management and symptom-based treatment such as medication in the form of anxiolytics or antidepressants (Yung et al, 1998). On the basis of these pilot data, they concluded that psychological interventions on their own may not be very successful in reducing transition to psychosis.

\section{Antipsychotics with and without psychotherapy}

This conclusion led them to use specific interventions as part of the first randomised controlled treatment trial to be published (McGorry et al, 2002) testing the impact of a specific intervention on psychosis transition rates. Fifty-nine help-seeking individuals aged 16-35 meeting their at-risk mental state criteria were randomly assigned to receive experimental or control treatments. Both experimental and control groups received a needs-based intervention that included case management, education and supportive psychotherapy targeted at the presenting symptom. In addition, the experimental group received medication (low-dose risperidone) combined with cognitive-behavioural therapy, interventions with known efficacy for full psychotic symptoms. The treatment phase lasted for 6 months, at the end of which there was a significant difference in rates of transition to full psychosis between the groups. Significance was lost after a further 6 months' follow-up, leading the authors to conclude that it is possible to delay, rather than prevent, the onset of psychosis.

The North American Prevention through Risk Identification, Management and Education (PRIME) study used closely similar entry criteria and opted to use an atypical antipsychotic (olanzapine) alone as the primary intervention (McGlashan et al, 2003b). This study was a double-blind randomised placebocontrolled trial. The intervention period lasted for 1 year, with individuals assessed for a further year without medication. This was a multi-site trial and randomised 60 people. At the 1-year analysis, 25\% of participants had made the transition to psychosis. Although the difference between the olanzapine and placebo groups was suggestive of an advantage of the drug treatment, it was not statistically significant (McGlashan et al, 2003a).

The use of antipsychotic medication in clients satisfying criteria for at-risk mental states is thought by some to be warranted. However, given the low accuracy of current assessment instruments and the implications of treating a large number of false positives, the side-effects of such medications are significant enough to consider alternatives (Bentall \& Morrison, 2002). Such side-effects, which commonly include weight gain and sexual dysfunction, can contribute to an individual's already high level of distress (French \& Morrison, 2004). In addition, in view of the age-group of the at-risk population (about 14-35 years), we must consider the implications of these issues on self-esteem and the effect that antipsychotic medication may have on the developing brain.

\section{Cognitive therapy alone}

A third trial, the Early Detection and Intervention Evaluation (EDIE) trial in the UK (Morrison et al, 2004), randomised 60 participants meeting Melbourne criteria to monitoring alone or to monitoring plus cognitive therapy. 
The Melbourne criteria were operationalised using the PANSS, distress levels measured with the General Health Questionnaire (GHQ) and/or recent deterioration in function assessed using the Global Assessment of Functioning (GAF).

Cognitive therapy was regarded as an acceptable and plausible treatment for a number of reasons. Cognitive therapy works with processes such as metacognitions and self-schemas, which are known to be abnormal in people at risk of psychosis. Birchwood et al (1989) showed that cognitive-behavioural monitoring of prodromal signs in clients with an existing diagnosis of psychosis enabled early intervention to prevent relapse or ameliorate mental state, and it has since been shown to significantly reduce relapse rates and hospital admissions in people at high risk of relapse (Gumley et al, 2003). In addition, cognitive therapy has been used in a number of randomised controlled trials involving individuals with acute and chronic psychotic symptoms, and has been shown to be efficacious (Drury et al, 1996; Kuipers et al, 1997; Tarrier et al, 1998; Sensky et al, 2000). Furthermore, most people in an at-risk mental state show significant affective symptoms, for which cognitive-behavioural therapy is an effective treatment in both anxiety disorders (e.g. Clark et al, 1999) and depression (e.g. Hollon et al, 1996). The importance that cognitive therapy places on developing shared problem lists and goals may also mean that it is a useful intervention for the false-positive group, who are seeking help for distressing symptoms but will not proceed to psychosis.

The trial found that transition rates according to PANSS threshold criteria were $12 \%$ at 1 year overall and were significantly lower in the cognitive therapy group than in the group that received monitoring alone (Morrison et al, 2004).

The EDIE trial has led to the delivery of local services in Salford, Manchester, under the name of the Early Detection and Intervention Team (EDIT).

Even cognitive therapy, however, raises ethical issues. People who turn out to have had a falsepositive result may be stigmatised by the experience, and therefore consideration should be given to the use of less pathologising language when dealing with clients. For example, labels such as prodromal, pre-psychotic and pre-schizophrenic could be replaced with distressed, help-seeking and at risk of developing psychosis (French \& Morrison, 2004).

\section{Levels of distress, help-seeking and Axis I disorders}

It is important to stress that the individuals included in the above trials were experiencing significant distress and disability, and were seeking help. It has been well documented by van Os and others that many people in the community have isolated psychotic experiences and are not distressed by them (van Os et al, 2000; Johns et al, 2004). On entry into the PACE clinic, participants had a mean GAF score of 61 , which equates to moderate levels of difficulty in social/role functioning (Yung et al, 2004). In the EDIE trial, the mean score of 49 showed greater impairment, and $85 \%$ of participants reached caseness levels on the GHQ (Morrison et al, 2004). Participants in the PRIME prevention study had a mean score of 42 on the GAF, which indicates a substantial level of functional disability (MacGlashan et al, 2003b). Importantly, there had been a mean drop of 15 points on this instrument over the year before entry into the study.

A significant proportion of those included in the trials discussed here also met criteria for a number of non-psychotic DSM-IV Axis I disorders. This alone qualifies them for receiving intervention of some kind. In Yung et al's (1996) pilot study, 86\% of their sample had symptoms of anxiety, $76 \%$ had depressed mood, $62 \%$ had low energy and $71 \%$ showed social withdrawal. In the prediction study carried out by McGorry et al (2002), of those who had not made the transition to psychosis at 12-month follow-up, $45 \%$ met criteria for mood and/or anxiety diagnoses.

\section{What predicts transition to psychosis?}

As previously stated, the PACE clinic reported that $28 \%$ of their sample had made the transition to psychosis by the 6-month assessment, rising to $35 \%$ at 12 months (Yung et al, 2004). On analysis of the baseline data they found significantly lower GAF scores (greater impairment) in those who subsequently made the transition. In comparing the length of time between the onset of 'symptoms' and time to first contact with appropriate services, they found that those in the transition group had also experienced significantly longer delays. Furthermore, those in the transition group had significantly higher levels of depression, measured with the Hamilton Rating Scale for Depression (HRSD). There were no significant differences between the groups on measures of anxiety, mania symptoms and negative symptom levels.

On completion of similar analyses on results in the EDIE trial, Morrison et al (2004) reported that multiple regression revealed the significant predictors of transition to be initial high PANSS positive scores and being in the control (monitoring alone) group rather than in the cognitive therapy group. 


\section{Who makes the referrals?}

Most of the individuals who took part in these and earlier first-episode studies had sought help from numerous sources before they reached appropriate services. In the Northwick Park first-episode psychosis study (Johnstone et al, 1986) clients trying to get help for their symptoms had an average of eight help-seeking contacts before receiving appropriate treatment. This situation contributes to the individual's distress and, in the UK, frequently ends in contact with the police and compulsory admission to hospital. There is some evidence that admissions under such circumstances may result in post-traumatic stress disorder (McGorry et al, 1991; Frame \& Morrison, 2001).

Phillips et al (1999) evaluated the referrals to the PACE clinic received over a 20 -month period. A sample of 45 people who met criteria and consented to take part were included in the analysis. The mean number of contacts with health or allied professionals was 2.3 (s.d. $=1.3$, range $1-7$ ). It was noted that first contacts had been with general health services or other professional services and that individuals gained access to mental health services only later. It was also noted that the average length of time between experiencing first symptoms and seeking help was 86 weeks. The average length of time between the first contact with healthcare or allied professionals and referral to PACE was 41.4 weeks. Therefore, the average time from onset of symptoms to contact with PACE was about $2 \frac{1}{2}$ years (127 weeks). The factors that the authors thought might have contributed to this over-long pathway to care (Box 2) suggest that education of both the public and general health services would play an important role in early detection and intervention in psychosis.

The majority of referrals to the PACE clinic came from a specialised early-psychosis assessment team known as the Youth Access Team (Phillips et al, 1999). This team has a variety of referral sources, including generic and mental health services, school and university counsellors, general practitioners,

Box 2 Factors that might delay access to earlyintervention services for young people

- The mildness of the symptoms

- The ego-syntonic nature of symptoms

- Failure of other services to refer on

- Lack of knowledge about such services

- Misinterpretation of symptoms

- Self-medication

- Fear of stigmatisation and labelling private psychologists/psychiatrists, and family and adolescent health services. The clinic also receives referrals directly from these sources. Sources of referrals to the EDIE trial included secondary mental health services, student health services, general practitioners, accident and emergency departments and community drug teams (Morrison et al, 2002).

This indicates that early detection and intervention services should spread their nets far and wide to reach the at-risk group. It also shows that there are many opportunities to provide training on at-risk mental states to individuals who come into contact with young people. Both those who have professional and specialist mental health backgrounds and those with little or no psychiatric knowledge could benefit from such as approach.

\section{Clinical guidelines for working with at-risk young people}

The International Early Psychosis Association has published advice regarding the early identification of psychosis (International Early Psychosis Association Writing Group, 2005). Young people who become less able to function or increasingly withdrawn, distressed or agitated for no apparent reason may be experiencing early psychotic phenomena. It suggests that individuals at risk of developing psychosis who are seeking help for distress or disability resulting from psychotic symptoms should be engaged and assessed and, if relevant, offered:

- regular monitoring

- interventions aimed at specific difficulties (e.g. anxiety, depression and substance misuse) and help with interpersonal, vocational and family issues

- psychoeducation and help to develop coping strategies for sub-threshold psychotic symptoms

- family support and education

- clear information about their risks of mental disorders, delivered in a careful and considered way.

Help should be delivered in surroundings that reduce stigmatisation, such as the individual's home, primary care settings or youth-based services.

\section{Conclusions}

The evidence suggests that it is possible both to identify individuals who may be at risk of developing psychosis and subsequently reduce or delay the transition to psychosis and ameliorate the severity of non-psychotic symptoms and distress. Specifically, 
cognitive therapy alone or in combination with lowdose atypical antipsychotics has been shown to be efficacious, whereas antipsychotic medication alone has not provided significant results. The highlighted ethical issues need to be considered seriously when working with young people thought to be at risk of developing psychosis, and further work is therefore needed to investigate the intervention options.

\section{Declaration of interest}

None.

\section{References}

Bentall, R. P. \& Morrison, A. P. (2002) More harm than good. The case against using antipsychotic drugs to prevent severe mental illness. Journal of Mental Health, 11, 351-365.

Birchwood, M., Smith, J., Macmillan, F., et al (1989) Predicting relapse in schizophrenia. The development and implementation of an early signs monitoring system using patients and families as observers. Psychological Medicine 19, 649-656.

Clark, D. M., Salkovskis, P. M., Hackmann, A., et al (1999) Brief cognitive therapy for panic disorder. A randomized controlled trial. Journal of Consulting and Clinical Psychology, 67, 583-589.

Crow, T. J., MacMillan, J. F., Johnson, A. L., et al (1986) The Northwick Park study of first episodes of schizophrenia. II: A controlled trial of prophylactic neuroleptic treatment. British Journal of Psychiatry, 148, 120-127.

Department of Health (2001) The Mental Health Policy Implementation Guide. London: TSO (The Stationery Office).

Department of Health (2003) Early Intervention for People with Psychosis: Expert Briefing. London: TSO (The Stationery Office).

Drury, V., Birchwood, M., Cochrane, R., et al (1996) Cognitive therapy and recovery from acute psychosis. I. Impact on psychotic symptoms. British Journal of Psychiatry, 169, 593601

Frame, L. \& Morrison, A. P. (2001) Causes of posttraumatic stress disorder in psychotic patients. Archives of General Psychiatry, 58, 305-306.

French, P. \& Morrison, A. P. (2004) Cognitive Therapy for People at High Risk of Psychosis. Chichester: John Wiley \& Sons.

Gumley, A. I., O'Grady, M., McNay, L., et al (2003) Early intervention for relapse in schizophrenia: results of a 12-month randomised controlled trial of cognitive behaviour therapy. Psychological Medicine, 33, 419-431.

Henquet, C., Krabbendam, L., Spauwen, J., et al (2005) Prospective cohort study of cannabis use, predisposition for psychosis, and psychotic symptoms in young people. BMJ, 330, 11 .

Hollon, S. D., DeRubeis, R. J. \& Evans, M. D. (1996) Cognitive therapy in the treatment and prevention of depression. In Frontiers of Cognitive Therapy (ed. P. M. Salkovskis), pp. 293317. New York: Guilford Press.

International Early Psychosis Association Writing Group (2005) International clinical practice guidelines for early psychosis. British Journal of Psychiatry, 187 (suppl. 48), s120-s124.

Johns, L. C., Cannon, M., Singleton, N., et al (2004) Prevalence and correlates of self-reported psychotic symptoms in the British population. British Journal of Psychiatry, 185, 298-305.

Johnstone, E. C., Crow, T. J., Johnson, A. L., et al (1986) The Northwick Park study of first episodes of schizophrenia. I: Presentation of the illness and problems relating to admission. British Journal of Psychiatry, 148, 115-120.

Klosterkotter, J., Hellmich, M., Steinmeyer, E. M., et al (2001) Diagnosing schizophrenia in the initial prodromal phase. Archives of General Psychiatry, 58, 158-164.

Kuipers, E., Garety, P., Fowler, D., et al (1997) London-East Anglia randomised controlled trial of cognitive-behavioural therapy for psychosis. I: Effects of the treatment phase. British Journal of Psychiatry, 171, 319-327.
Leicester, S. (2006) Substance use and the 'at risk' period. In Working with People at High Risk of Developing Psychosis: A Treatment Handbook (eds J. Addington, S. Francey \& A. P. Morrison), pp. 93-110. Chichester: John Wiley \& Sons.

Loebel, A. D., Lieberman, J. A., Alvir, J. M. J., et al (1992) Duration of psychosis and outcome in first episode schizophrenia. American Journal of Psychiatry, 149, 1183-1188.

McGlashan, T., Miller, T., Woods, S. W., et al (2001) A scale for the assessment of prodromal symptoms and states. In Early Intervention in Psychotic Disorders (eds T. Miller, S. A. Mednick, T. H. McGlashan, et al), pp. 135-150. Dordrecht: Kluwer Academic Publishing.

McGlashan, T., Zipursky, R. B., Perkins, D., et al (2003a) Olanzapine versus placebo treatment of the schizophrenia prodrome. One year results. Schizophrenia Research, 60 (suppl. 1), 295.

McGlashan, T. H., Zipursky, R. B., Perkins, D., et al (2003b) The PRIME North America randomized double-bind clinical trial of olanzapine versus placebo in patients at risk of being prodromally symptomatic for psychosis. I. Study rationale and design. Schizophrenia Research, 61, 7-18.

McGorry, P. D., Chanen, A., McCarthy, E., et al (1991) Post traumatic stress disorder following recent onset psychosis. Journal of Nervous and Mental Disease, 179, 253-258.

McGorry, P. D., Yung, A. R., Phillips, L. J., et al (2002) Randomized controlled trial of interventions designed to reduce the risk of progression to first-episode psychosis in a clinical sample with subthreshold symptoms. Archives of General Psychiatry, 59, 921-928.

Miller, P., Lawrie, S. M., Hodges, A., et al (2001) Genetic liability, illicit drug use, life stress and psychotic symptoms: preliminary findings from the Edinburgh study of people at high risk for schizophrenia. Social Psychiatry and Psychiatric Epidemiology, 36, 338-342.

Miller, T. J., McGlashan, T. H., Wood, S. W., et al (1999) Symptom assessment in schizophrenic prodromal states. Psychiatric Quarterly, 70, 273-287.

Morrison, A. P., Bentall, R. P., French, P., et al (2002) Randomised controlled trial of early detection and cognitive therapy for preventing transition to psychosis in high risk individuals. Study design and interim analysis of transition rate and psychological risk factors. British Journal of Psychiatry, 181 (suppl. 43), s78-s84.

Morrison, A. P., French, P., Walford, L., et al (2004) Cognitive therapy for the prevention of psychosis in people at ultra-high risk. Randomised controlled trial. British Journal of Psychiatry, 185, 291-297.

Phillips, L., Yung, A. R., Hearn, N., et al (1999) Preventative mental health care: accessing the target population. Australian and New Zealand Journal of Psychiatry, 33, 912-917.

Phillips, L. J., Yung, A. R. \& McGorry, P. D. (2000) Identification of young people at risk of psychosis: validation of Personal Assessment and Crisis Evaluation Clinic intake criteria. Australian and New Zealand Journal of Psychiatry, 34 (suppl.) S164-S169.

Phillips, L. J., Curry, C., Yung, A. R., et al (2002) Cannabis use is not associated with the development of psychosis in an 'ultra' high-risk group. Australian and New Zealand Journal of Psychiatry, 36, 800-806.

Sensky, T., Turkington, D., Kingdon, D., et al (2000) A randomized controlled trial of cognitive-behavioral therapy for persistent symptoms in schizophrenia resistant to medication. Archives of General Psychiatry, 57, 165-172.

Tarrier, N., Yusupoff, L., Kinner, C., et al (1998) A randomized controlled trial of intense cognitive behaviour therapy for chronic schizophrenia. BMJ, 317, 303-307.

van Os, J., Hanssen, M., Bijl, R. V., et al (2000) Strauss (1969) revisited: a psychosis continuum in the normal population? Schizophrenia Research, 45, 11-20.

Verdoux, H., Gindre, C., Sorbara, F., et al (2003) Effects of cannabis and psychosis vulnerability in daily life. An experience sampling test study. Psychological Medicine, 33, 23-32.

Warner, R. (2002) Limitations of the Bonn Scale for the Assessment of Basic Symptoms as a screening measure. Archives of General Psychiatry, 59, 5.

Wyatt, R. (1991) Neuroleptics and the natural course of schizophrenia. Schizophrenia Bulletin, 17, 325-351. 
Yung, A., McGorry, P. D., McFarlane, et al (1996) Monitoring and care of young people at incipient risk of psychosis. Schizophrenia Bulletin, 22, 283-303.

Yung, A., Phillips, L. J., McGorry, P. D., et al (1998) A step towards indicated prevention of schizophrenia. British Journal of Psychiatry, 172 (suppl. 33), 14-20.

Yung, A., Phillips, L. J., McGorry, P. D., et al (2001) Comprehensive Assessment of At-Risk Mental States (CAARMS). Melbourne: PACE Clinic, University of Melbourne.

Yung, A. R., Phillips, L. J., Yuen, H. P., et al (2003) Psychosis prediction: 12-month follow up of a high-risk ('prodromal') group. Schizophrenia Research, 60, 21-32.

Yung, A. R., Phillips, L. J., Yuen, H. P., et al (2004) Risk factors for psychosis in an ultra high-risk group: psychopathology and clinical features. Schizophrenia Research, 67, 131-142.

\section{MCQs}

1 People are thought to be at risk of developing psychosis if:

a they have a relative with a diagnosis of a psychotic disorder

b they experience subclinical symptoms, which remit within 1 week

c they experience frank psychotic symptoms, which remit within 1 week

d they meet criteria for schizotypal personality disorder

e their ability to function has deteriorated in the previous 2 months.

2 The following has been shown to be effective in reducing the risk of developing psychosis in at-risk mental states:

a antipsychotic medication

b cognitive therapy alone or in conjunction with lowdose atypicals

c antidepressant drug treatment

d nothing

e monitoring alone.
3 Individuals thought to be at risk of developing psychosis have sought help from the following:

a GPs

b school counsellors

c accident and emergency departments

d community drugs teams

e all of the above.

4 Participants of trials investigating at-risk mental states:

a were asking for help and experiencing significant distress

b were not asking for help

c were not distressed

d did not meet criteria for mood diagnoses

e did not meet criteria for anxiety diagnoses.

5 Transition to psychosis is most strongly predicted by high levels of:

a anxiety

b mania

c negative symptoms

d positive symptoms

e functioning.

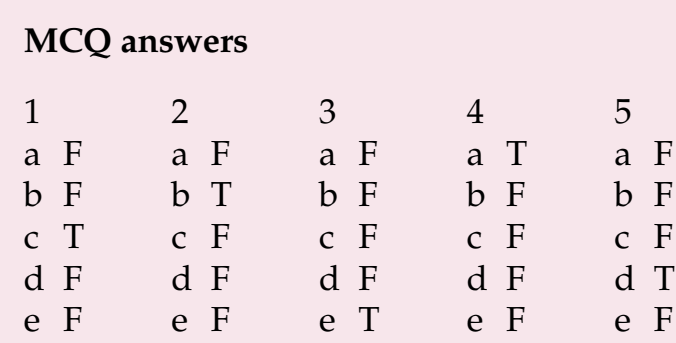

